This is a post-print version of the following article: Hernández, M.I., Couso, D., Pintó, R. (2012). The analysis of students' conceptions as a support for designing a teaching/learning sequence on the Acoustic Properties of Materials. Journal of Science Education and Technology, 21 (6), 702-712.

DOI: $10.1007 / \mathrm{s} 10956-011-9358-4$

https://link.springer.com/article/10.1007\%2Fs10956-011-9358-4\#page-1

\title{
The analysis of students' conceptions as a support for designing a teaching/learning sequence on the Acoustic Properties of Materials
}

\author{
María Isabel Hernández, Digna Couso and Roser Pintó
}

Centre for Research in Science and Mathematics Education (CRECIM), Universitat Autònoma de Barcelona (UAB), Campus de Bellaterra, GL-304, 08193, Cerdanyola, Barcelona, Spain

e-mail: mariaisabel.hernandez@uab.es

\begin{abstract}
We believe that finding out how students think about certain topics that are covered in science classes should not be "the end of the story" but the starting point for planning lessons and designing materials. From this perspective, the research study presented here is intended to explore secondary school (15-18 year old) students' preconceptions of sound attenuation, and of the properties and internal structure of materials. Specifically, we analysed students' explanations for the fact that some materials attenuate sound more than others. This study was conducted within a particular scenario, in which 72 students participated in laboratory sessions aimed at developing students' understanding of the nature, propagation and attenuation of sound. From the analysis of students' explanations, we could identify some conceptions of sound attenuation in materials (e.g. as a result of hindering the entrance of sound, or as a result of capturing sound). The results of this study also indicate that the role of properties of a material and the role of the internal structure that students associate with its acoustic behaviour depend on their conceptions of sound attenuation. We used these results as support for the design of a research-based teaching/learning sequence on the Acoustic Properties of Materials, which is intended to facilitate students' overcoming the specific conceptual difficulties identified in this research study and promote students' development of conceptual models of sound attenuation.
\end{abstract}

\section{Keywords}

Sound attenuation, Acoustic properties of materials, Research-based design, Students' conceptions, Teaching/learning sequence, Secondary school

\section{Introduction}

It has been widely evidenced that students develop ideas about natural phenomena before they are taught science in school. It is also widely agreed that such ideas should not be seen as simply pieces of misinformation, but as students' ways of constructing events and phenomena which can be coherent and fit with their domains of experience, even though they may differ substantially from the scientific view. We agree with Driver, Squires, Rushworth, and Wood-Robinson (1994) that finding out how students think about the various topics covered in science classes is not "the end of the story" (p. 10). Science teachers have the responsibility for providing activities which enable students to make the journey from their current understanding of the world to the accepted scientific view.

There is a wide consensus in science education that effective science teaching takes account of students' previous ideas, among other aspects. According to this view, this article presents a research study about students' preconceptions of a specific topic - sound attenuation in materials and the acoustic properties of materials - in order to provide new insights into how to teach this topic in secondary school.

Even though several research studies have analysed students' conceptions of the nature of sound, sound propagation and other sound-related phenomena such as resonance or superposition, we could not find previous published studies specifically devoted to the analysis of students' conceptions of sound attenuation in materials and the acoustic properties of materials. That was one of the main reasons to conduct this study. 
Another reason for conducting this research relates to the particular context in which we planned to carry out the study. According to the available research data which supports that students' thinking is deeply situated in specific contexts (Taber 2000), we assumed that the students' responses that might be obtained in the particular scenario we used (understood as the setting and the activities) would presumably be different (and even richer and more varied) from students' responses to the same questions had they been asked before having performed some previous activity related to the same phenomenon (sound attenuation). Multiple possible contexts can be used to explore children's thinking about sound attenuation, but we selected a (technologically and pedagogically) rich scenario in which students were asked about their thinking of sound attenuation referring to one experimental task that they had previously performed during a laboratory session.

In particular, the research we present here was aimed at contributing to the design of a teaching/learning sequence (TLS) on the Acoustic Properties of Materials (APM) intended for 15-16 year-old secondary school students. Thus, this article will report on some of the previous steps leading up to the design of the TLS on APM, which was carried out after the research reported here and took its findings into account.

First of all, this paper presents a review of previous studies of students' conceptions of sound reported in the literature. Then, it explains the research study we carried out on students' preconceptions of the specific topics we wanted to address in the sequence - sound attenuation and the acoustic properties of materials. Finally, the article describes the findings of this research and how they were used to support the design of the teaching sequence on APM.

\section{Students' conceptions of sound before or after formal instruction}

As mentioned before, one of the first actions carried out when designing a TLS on APM was to consider the ideas about sound usually held by secondary school students, and the difficulties they commonly have in understanding what sound is, how it propagates and how it is attenuated. According to Wittmann, Steinberg, and Redish (2003), whilst the concepts of sound and noise are part of students' everyday experience, the fact that the perception of sound is auditory and not visual makes knowing what sound is, how it propagates and how it is attenuated become even more difficult for many students.

From a scientific point of view, sound is conceived as the event produced by the vibrations of an object called the sound source. These vibrations are propagated through an elastic medium which gradually transmits its state of compression or dilation, without transport of matter. The important point here is that sound, as a wave, is a process of energy transfer and therefore has the physical properties of processes, not those of objects (Lawrence 2008). Most of the research studies devoted to analysing students' conceptions of sound concluded that this key idea is not understood by most students.

Below, we summarise the main findings of several previous studies on students' common conceptions of sound, before or after formal instruction, distinguishing two categories: conceptions referring to what sound is (i.e. ontology attributes) and how it propagates (mechanisms and trajectory), and conceptions referring to the interaction between sound and the medium through which it propagates.

\subsection{Students' conceptions of the nature and propagation of sound}

Several studies suggest a naïve mental model of sound propagation, according to which sound travels as a particle-like object. This kind of mechanistic spontaneous reasoning has been evidenced in elementary school students (Mazens \& Lautrey 2003), as well as in secondary school students (Eshach \& Schwartz 2006; Maurines 1993) and undergraduate physics students (Hrepic, Zollman \& Rebello 2010; Linder 1992; Wittmann et al. 2003). Some of these authors generally refer to this naïve model as the "particle model" (Maurines, 1993) or "entity model" (Hrepic et al. 2010) of sound propagation. These models can be characterised in terms of some of the following attributes expressed by students in the aforementioned studies:

- Sound signals are conceptualised as material objects (sound particles) created and set in motion by the source (Maurines 1993).

- Sound is considered an entity which is transported by individual molecules, which move along a medium (Linder 1992).

- Sound is considered an entity which is transferred from one molecule of a medium to another but is different from the medium where it propagates (Linder 1992). 
- Sound is considered a limited substance which travels with a certain impetus, and is generally represented as an air current (Linder 1992).

- Sound is considered a substance which travels following the pattern of waves (Linder 1992).

\subsection{Students' conceptions of the interaction of sound with matter}

Some research studies (Linder 1993; Maurines 1993) also analysed students' explanations of the factors that affect the speed of sound, and students' ideas about the interaction of sound with a certain medium. Here we summarise the main findings that relate to our study:

- Molecules that form a medium are conceptualised as an obstacle to the propagation of sound through the medium (Linder 1993).

- The speed of sound is conceived as being dependent on the source or the signal amplitude but independent of the properties of the medium (Maurines 1993).

- Even when the speed of sound is recognised as being dependent on the density and elasticity of materials, density is often conceptualised as related only to the distance between the molecules of a medium, and elasticity is conceptualized in terms of compressibility and as inversely proportional to density (Linder 1992).

- Sound is thought to be able to propagate through the vacuum and to be transmitted through the empty spaces between the particles that form a medium (Maurines 1993).

To sum up, these research studies indicate that previous knowledge regarding sound of students at any educational level tends to be materialistic or "based on substances". This implies that students tend to attribute properties or the behaviours of material substances to processes or events, as in the case of sound. On the other hand, the previous studies evidence that students often attribute different roles to the medium when referring to sound propagation (facilitating or hindering sound propagation) and, moreover, the physical properties of the medium such as density and elasticity are themselves difficult to be appropriately conceptualised.

Although many aspects and attributes of the so-called "entity model" of sound have been described and reported by several authors, these previous research studies did not tackle the analysis of how students conceive sound attenuation or how they understand the acoustic properties of materials. For this reason, we decided to design and conduct a research study to explore 15-18 year-old students' preliminary ideas of these topics. This research study was carried out within a special scenario which is described below.

\section{Context of the study}

\subsection{The REVIR scenario}

The scenario in which this study was conducted is the REVIR project, which is an initiative of the Centre for Research in Science and Mathematics Education (CRECIM), in which secondary school students from Catalonia can access a computerised laboratory at the University (Faculty of Education). Groups of researchers and secondary school teachers collaborate to design activity sequences for labwork practice that deal with different science topics and integrate different ICT tools (simulations, computational applications and MBL technology). The aim for including these ICT tools is not only for improved learning of science content and skills, but also a greater achievement of digital competence and a more sophisticated idea of how real science is conducted in ICT-enhanced laboratories (Pintó, Couso \& Hernández 2010).

During the REVIR sessions, classes of secondary school students (12-18 years old) spend an entire morning (4 hours) working in small groups (2-4 students) with specific material prepared for the session and for the grade of the attending class. Their teachers also attend the session in order to observe their students' attitudes and skills when working with computational tools and, at the same time, they learn different ways of implementing these tools within their own lessons. Members of CRECIM implement the designed sequences and often collect data about the learning process. In short, the REVIR project is a hybrid scenario intended to contribute to both science teaching and research in science education. 
The teaching sequence on Acoustics education ${ }^{1}$ addressed to these REVIR sessions was designed and iteratively developed for several months before the data collection for the study we present here took place. This teaching sequence engages students in analysing the nature, propagation, production and hearing of sound, in monitoring and characterising the sounds they produce and in designing solutions to attenuate sound, the content of which is included in the official "Physics and Chemistry" curriculum.

\subsection{The teaching sequence on Acoustics education for the REVIR scenario}

As a method to involve students in the session, the teaching sequence begins by placing students in a real context with some significance to them, and by posing a meaningful problem. The aim is to give them a purpose for the tasks that they will carry out during the session, challenging them to find a solution to the following problem:

We are a rock team practising in the attic of a building. While we were practising, the police came due to some complaints from the neighbours. We must attenuate the sound we produce by using some materials. How can we manage to do it?

The teaching sequence on Acoustics education is structured around three tasks:

a) Let's explore and describe sound

Students explore a simulation ${ }^{2}$, which represents the propagation of sound in terms of the vibration of the particles that form a medium, and also graphically represents the displacement of particles over time. Students are asked a series of questions that are intended to guide them when exploring the simulation about sound and its characteristics. The goal of this first task is that students revise/clarify their conceptions of sound propagation in terms of the vibrations of particles and the transfer of energy but not matter. Throughout the task, students discuss the meaning of the variables that characterise sound waves (frequency, period, amplitude) to guarantee that they share a specific language with a common meaning during the session.

\section{b) Let's graphically analyse different sounds and noises}

Students analyse the graphical representations of different real sounds by means of a freeware application $^{3}$ to characterise sound waves produced by different instruments in terms of their frequency and shape, related to the pitch and timbre respectively. Therefore, students relate the pitch of different sounds to the frequencies they calculate from the period expressed in the graphs, and they relate the shape of the graphs to the characteristic timbre of an instrument. Students also graphically analyze the difference between sound and noise. In short, students analyse in depth one of the variables that characterise sound: frequency (or period).

\section{c) Let's measure sound intensity level}

In this final task, students focus on another key variable that characterises sound waves: the amplitude. First of all, students relate the amplitude of sound waves to the sound intensity level, magnitude that they learn to measure with a sound level meter. Next, students design and perform an experiment to compare the capacity for attenuating sound with three different materials: expanded polystyrene, glass wool and rock wool. At this point, the only operational definition of sound attenuation that is made explicit and shared among students is that sound attenuation implies a decrease in sound intensity level. Although this activity engages students in analysing the acoustic behaviour of different materials, this activity is not intended to engage students in discussing or analysing the properties of materials that influence their acoustic behaviour ${ }^{4}$. Once the students perform the experiment and obtain the resulting measurements, they are expected to make informed decisions on the material they would propose as soundproofing solutions for the noise control of the room of the problem posed at the start of the session.

\subsection{The experiment to analyse materials' capacity for attenuating sound}

\footnotetext{
${ }^{1}$ Note that the teaching sequence on Acoustics education designed for the REVIR sessions is different from that on the Acoustic Properties of Materials (APM) which will be described later and which was designed using the results obtained from the present research study.

${ }^{2}$ http://www.ngsir.netfirms.com/englishhtm/Lwave.htm

${ }^{3}$ Audacity (http://audacity.sourceforge.net)

${ }^{4}$ This is indeed the main focus of the teaching/learning sequence on the Acoustic Properties of Materials which was designed using the results obtained from the current research study
} 
The experimental setup used in the investigation carried out by the students in the third task of the teaching sequence contains the following elements: a sound source, samples of material and a detector.

Regarding the sound source, students are provided with broadband buzzers, which emit sound at a constant intensity level.

The samples or plates of material are contained inside a cardboard box covering all six sides. The cardboard box itself represents the structure of a room or closed space, which has a particular geometry. The plates of material covering the box (as shown in Figure 1) represent the samples of the material that could be used to soundproof a certain room. These samples have similar thickness $(2.5 \mathrm{~cm})$ to allow comparison of the capacity of the different materials for attenuating sound, instead of the effect due to the different thicknesses of the plates. Three boxes were used in order to cover each of them with plates made from a different material.

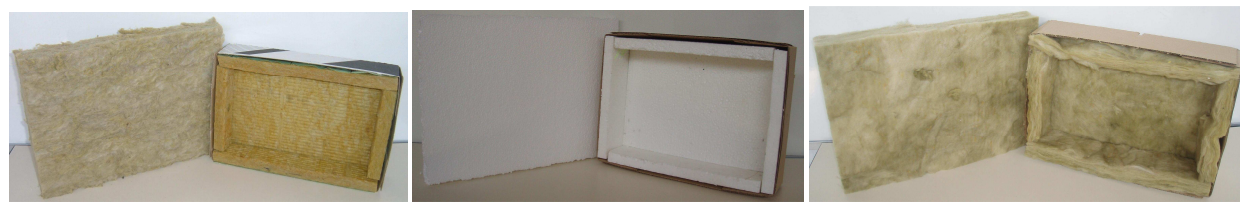

Fig. 1 Cardboard boxes and their lids covered with rock wool, expanded polystyrene and glass wool, respectively

As a detector, students use sound level meters to measure the sound intensity level, which are the common instruments used in everyday life for noise control. Sound level meters are also used in connection with data capture systems (MBL technology) and computers (Figure 2) in order to register and monitor in real time the evolution of the sound intensity level during the experiments.

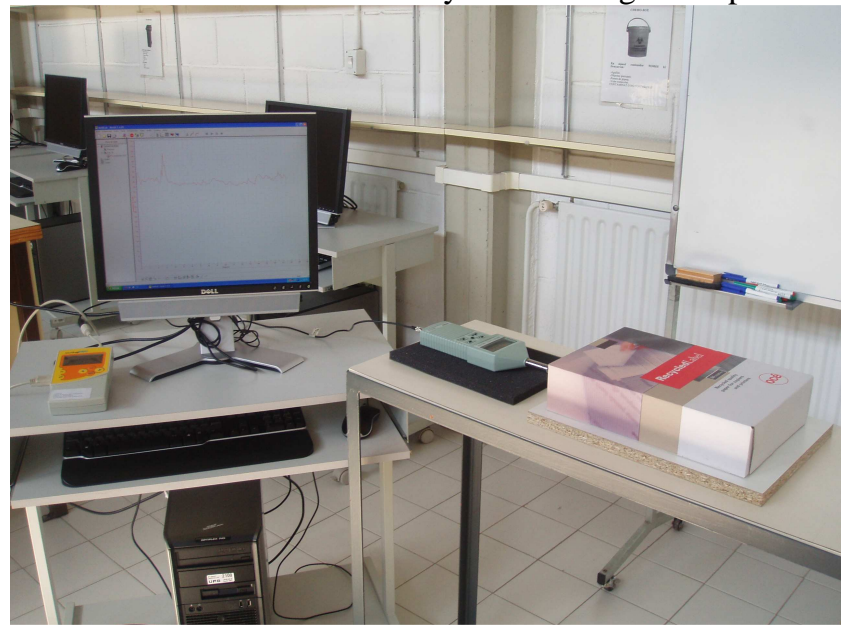

Fig. 2 Experimental setup (sound level meter-MBL interface-computer, box and lid covered with a certain material, buzzer inside the box)

During the experiment, students use the sound level meter to measure the intensity level of the sound transmitted outside a box whose walls have been covered with plates made from a certain material (sample), having placed the sound source inside the box (Figure 3). The measurement of the intensity level of the sound transmitted outside the box covered with the tested material represents the noise control measurement that would be taken at the neighbours' house. Comparing the measurements outside each box at a certain (fixed) distance from the source, students can compare the capacity of each material for attenuating sound, with the lowest measurement outside the box being associated with the best sound insulating material or with the one that has higher capacity for attenuating sound.

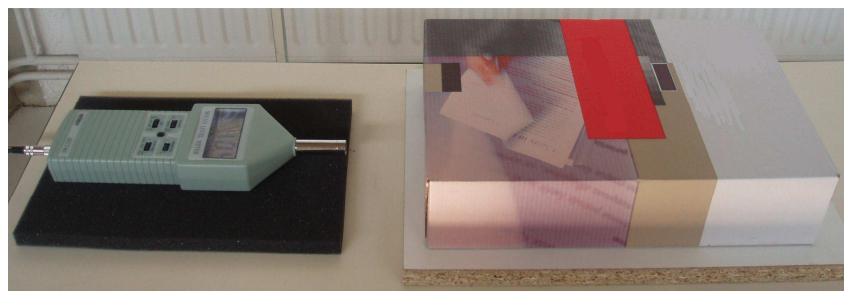


Fig. 3 Experiment to test sound attenuation produced by the material inside the box

After describing in detail the intervention carried out during the REVIR sessions devoted to Acoustics education, in which the research data were collected, the specific research questions and methods are discussed.

\section{Research questions}

In designing our study, we were guided by two research questions: (a) What preconceptions on the phenomenon of sound attenuation do secondary school students use when explaining the acoustic behaviour of materials? (b) Which properties and internal structure do secondary school students assign to materials according to their acoustic behaviour?

\section{Method}

Setting and Participants

Seventy-two secondary school students from different Catalan schools constitute the sample of our study. These students came from different classes that participated in REVIR sessions that dealt with Acoustic education throughout two consecutive academic years (2006-07, 2007-08). The participating students were studying in the final years of secondary school (15-18 years old). Although all the participating students had, to some extent, previously studied the topic of sound during their formal science instruction, they had not formally discussed the topic of sound attenuation in materials and the acoustic properties of materials.

Data Sources

Our methodological choice consisted of an open-ended questionnaire administered at the end of the entire REVIR session on Acoustics education, after students performed the experiment already described, and without having discussed the mechanisms of sound attenuation or acoustic properties of the materials used during the session. For the purpose of this research, students were asked the following open-ended question concerning the third task of the session:

Invent an explanation for the fact that some materials tested attenuate sound more than other materials

\section{Data Analysis}

We conducted a phenomenographic study using a qualitative research methodology within the interpretative paradigm, in order to investigate the different ways in which students experience something or think about a specific situation or phenomenon (Marton 1981). Thus, this phenomenographic study was intended to obtain a set of related categories that describe the qualitative differences between one students' conception and another.

\section{Results and Discussion}

Analysing students' answers, we could categorise students' explanations depending on whether they focused on the acoustic behaviour of different materials or on the physical properties or internal structure of materials. Table 1 summarises the different types of students' explanations that were identified.

Table 1. Types of students' explanations (at the end of the session) for the fact that some tested materials attenuate sound more than others

\begin{tabular}{|l|l|l|c|}
\hline $\begin{array}{c}\text { Explanation } \\
\text { in terms of... }\end{array}$ & \multicolumn{1}{|c|}{$\begin{array}{c}\text { Description of each type of } \\
\text { explanation }\end{array}$} & \multicolumn{1}{|c|}{ Students' quotes } & $\begin{array}{c}\text { Number of } \\
\text { students }\end{array}$ \\
\hline $\begin{array}{l}\text { Acoustic } \\
\text { Behaviour of } \\
\text { Materials } \\
(A B M)\end{array}$ & $\begin{array}{l}\text { Students explain the different } \\
\text { capacities for attenuating sound of } \\
\text { different materials by providing a } \\
\text { description of the acoustic behaviour of } \\
\text { materials }\end{array}$ & $\begin{array}{l}\text { "[Some materials attenuate } \\
\text { sound more than others] } \\
\text { because they do not allow } \\
\text { the passage of sound waves" }\end{array}$ & $39 / 72$ \\
\hline Physical & Students interpret the differences in & "[Some materials attenuate & $63 / 72$ \\
\hline
\end{tabular}




\begin{tabular}{|c|c|c|c|}
\hline $\begin{array}{l}\text { Properties of } \\
\text { Materials } \\
(P P M) \\
\end{array}$ & $\begin{array}{l}\text { sound attenuation produced by } \\
\text { different materials in terms of their } \\
\text { physical properties }\end{array}$ & $\begin{array}{l}\text { sound more than others] } \\
\text { because they are less } \\
\text { porous" }\end{array}$ & $(88 \%)$ \\
\hline $\begin{array}{l}\text { Internal } \\
\text { Structure of } \\
\text { Materials } \\
(I S M) \\
\end{array}$ & $\begin{array}{l}\text { Students relate the different capacities } \\
\text { for attenuating sound of different } \\
\text { materials to their internal structure in } \\
\text { terms of the particulate model of matter }\end{array}$ & $\begin{array}{l}\text { "[Some materials attenuate } \\
\text { sound more than others] } \\
\text { because their particles are } \\
\text { more distant [...]" }\end{array}$ & $\begin{array}{c}8 / 72 \\
(11 \%)\end{array}$ \\
\hline
\end{tabular}

As shown in Table 1, many students (54\%) explained the differences in sound attenuation caused by different materials by providing a description of the acoustic behaviour of these materials $(A B M)$. Thus, these students made explicit what they understand by sound attenuation.

Most of the students (88\%) also explained possible differences in the acoustic behaviour of materials in terms of their physical properties $(P P M)$. That is to say that students mentioned several properties influencing the particular acoustic behaviour of each material, perhaps not even eliciting their idea of sound attenuation.

We surprisingly found that $11 \%$ of students, without having been taught about the role of internal structure of a material, used a microscopic model (ISM) to justify their capacity for attenuating sound.

The previous percentages already provided evidence that in many cases students did not answer in terms of just one type of the aforementioned explanations but in terms of a combination of some of them. For example, the next student's quote illustrates an explanation that refers to a physical property of materials $(P P M)$, the internal structure of matter (ISM), and also makes explicit the acoustic behaviour of materials $(A B M)$ :

"If a material is denser, its particles are closer, and therefore it transmits sound better than a less dense material"

We now proceed to analysing in depth and separately the types of explanations that students formulated when are asked about sound attenuation. The $A B M$-type students' explanations were analysed in order to answer the first research question, and the PPM and ISM-types students' explanations were analysed to answer the second research question. The content analysis of these students' explanations is described below.

\subsection{Students' conceptions of sound attenuation}

In order to identify students' preconceptions of sound attenuation, we took a subsample of 39/72 students (54\%), which is formed from those who have answered in terms of the acoustic behaviour of materials $(A B M)$. Table 2 shows such preconceptions.

Table 2. Description of students' conceptions of sound attenuation

\begin{tabular}{|c|l|l|c|}
\hline $\begin{array}{c}\text { Student } \\
\text { conception }\end{array}$ & \multicolumn{1}{|c|}{$\begin{array}{c}\text { Description of the } \\
\text { conception }\end{array}$} & \multicolumn{1}{c|}{ Students' quote } & $\begin{array}{c}\text { Number of } \\
\text { students }\end{array}$ \\
\hline $\mathbf{C 1}$ & $\begin{array}{l}\text { Sound is attenuated by } \\
\text { hindering the entrance of } \\
\text { sound }\end{array}$ & $\begin{array}{l}\text { "Depending on the properties of materials, } \\
\text { they allow the passage of waves more or } \\
\text { less. The materials that best insulate } \\
\text { sound, reflect waves more" }\end{array}$ & $\begin{array}{c}11 / 39 \\
(28 \%)\end{array}$ \\
\hline $\mathbf{C 2}$ & $\begin{array}{l}\text { Sound is attenuated while } \\
\text { propagating within/ } \\
\text { through a material }\end{array}$ & $\begin{array}{l}\text { "The denser a material is, the less } \\
\text { vibration it allows in its particles, and } \\
\text { therefore it has more difficulty } \\
\text { transmitting sound" }\end{array}$ & $\begin{array}{c}15 / 39 \\
(38 \%)\end{array}$ \\
\hline $\mathbf{C 3}$ & $\begin{array}{l}\text { Sound is attenuated by } \\
\text { capturing / absorbing } \\
\text { sound within the material }\end{array}$ & $\begin{array}{l}\text { "Some materials absorb the vibration } \\
\text { [...]more easily than others because they } \\
\text { have small spaces full of air" }\end{array}$ & $\begin{array}{c}7 / 39 \\
(18 \%)\end{array}$ \\
\hline & $\begin{array}{l}\text { Sound is attenuated by } \\
\text { either reflecting or } \\
\text { absorbing sound }\end{array}$ & $\begin{array}{l}\text { "Some materials have pores that absorb } \\
\text { sound; } \text { others do not have [any pore] and } \\
\text { reflect sound" }\end{array}$ & $\begin{array}{c}6 / 39 \\
(15 \%)\end{array}$ \\
\hline
\end{tabular}

As summarised in Table 2, more than one quarter of the reduced sample of students considers that sound attenuating materials behave as sound barriers that prevent the passage of sound through a material (C1). Accordingly, these students conceive that the only mechanism of sound attenuation is sound reflection. 
Students' conception of sound attenuation C1 seems to be based on an underlying conception of sound as a physical entity that can or cannot move through a material depending on certain characteristics of the material. Even in the case of students explicitly associating the propagation of sound with energy transfer they might consider sound and/or energy as a substance that can propagate or be obstructed by certain materials.

"[Some materials attenuate sound more than others] because there are materials that allow the energy, which is transmitted by sound by means of the particles [of the medium], to go through them more than other materials"

Therefore, as many authors evidenced in several previous research studies (Maurines 1993, Hrepic et al. 2010), the concept of sound as an entity instead of as a process is rather common.

As also illustrated in Table 2, most of the students (38\%) accounted for sound attenuation in materials as being the difficulty for sound to be transmitted or the difficulty for particles of the medium to vibrate (C2). Students often associate this difficulty with certain modifications to the characteristics of sound waves. One of the changes to sound waves that students highlight is the "decrease" in energy, as exemplified by the following quote:

"The most porous materials are the most sound insulating materials because when a wave finds

obstacles [within the material] it loses energy, and therefore the sound loses intensity"

Some of the students that hold this conception C2 argue that sound transmission through different solid materials is more or less difficult depending on the distance between the particles of each material. The following student's quote evidences this type of conception:

"[Some materials attenuate sound more than others] because their particles are more separated

and the vibration is transmitted worse than in a material whose particles are very close"

According to Linder (1993), students generally consider that the distance between particles affects the speed of sound propagation, so that the smaller the intermolecular distance the faster the sound travels in the material. Students interpret that individual molecules of the material have to travel less distance to transfer sound to the next molecule. We interpret that students answer in terms of difficulty in sound transmission since they might conceive sound attenuation as a decrease in the speed of sound propagation in a medium. This conception would imply that the more sound is attenuated, the slower it propagates through a medium.

Remarkably, about $20 \%$ of students recognised sound absorption as a phenomenon that accounts for sound attenuation (C3), even though absorption was not explained in terms of energy dissipation. In some cases, students who explained sound attenuation as the absorption of sound within a material also evidenced a materialistic reasoning in terms of the entity or particle model of sound. For instance, one student answered:

"If there is more material inside the box, it holds sound more, and thus, sound is heard less"

According to the previous answer, sound is conceived as an entity that can be held or kept within the material.

Finally, it is worth noting that $15 \%$ of students considered both reflection and absorption as mechanisms of sound attenuation (C4), although in most of the cases they did not explain what they meant by absorption or relate it to energy dissipation.

\subsection{Students' conceptions of acoustic properties of materials}

From the subsample of students $(66 / 72,92 \%)$ that explained the differences in materials' capacity for attenuating sound in terms of their physical properties and/or internal structure (PPM and/or ISM respectively), we determined the characteristics that students associate with the acoustic behaviour of materials (Table 3 ).

Table 3. Characteristics that students associate with the acoustic behaviour of materials

\begin{tabular}{|c|c|c|c|}
\hline \multicolumn{2}{|c|}{$\begin{array}{c}\text { Materials' capacity for attenuating sound } \\
\text { is associated with ... }\end{array}$} & Characteristic of materials & Number of students \\
\hline $\begin{array}{c}\text { Physical } \\
\text { properties of } \\
\text { materials }\end{array}$ & $\begin{array}{c}\text { Intensive } \\
\text { (scale invariant) }\end{array}$ & Porosity & $32 / 66(48 \%)$ \\
\cline { 2 - 3 } & Density & $29 / 66(44 \%)$ \\
\cline { 2 - 3 }
\end{tabular}




\begin{tabular}{|c|c|c|c|}
\hline \multirow[t]{3}{*}{$(P P M)$} & \multirow{3}{*}{$\begin{array}{c}\text { Extensive } \\
\text { (dependent on the system } \\
\text { size or the amount of } \\
\text { material) }\end{array}$} & Thickness & $5 / 66(8 \%)$ \\
\hline & & Number of layers & $4 / 66(6 \%)$ \\
\hline & & Quantity of matter & $5 / 66(8 \%)$ \\
\hline \multirow{2}{*}{\multicolumn{2}{|c|}{$\begin{array}{l}\text { Internal structure of materials } \\
\qquad(I S M)\end{array}$}} & Distance between particles & $5 / 66(8 \%)$ \\
\hline & & Arrangement of particles & $1 / 66(2 \%)$ \\
\hline
\end{tabular}

As illustrated in Table 3, students mentioned several characteristics of materials as influencing their acoustic behaviour. The richness and variety of characteristics of materials mentioned by students is worth noting since they come from their inferences after the manipulation and observation of the three sound attenuating materials used in the experiment (rock wool, expanded polystyrene and glass wool). In particular, most of the students explained the differences in the materials' capacity for attenuating sound in terms of intensive properties, such as porosity, density, and hardness. Taking into account that porosity, density and elasticity are in fact the most relevant acoustic properties of materials (Hernández, Couso \& Pintó 2011), this result is quite remarkable.

Furthermore, a sample of 33/72 (46\%) students established relationships between certain physical properties or the internal structure of materials (categories PPM or ISM in Table 1) and acoustic behaviour of materials ( $A B M$ in Table 1). From these responses, we can determine that each property is conceived by different students as affecting the materials' capacity for attenuating sound in different ways. Table 4 presents the different roles attributed to different properties.

Table 4. Relationships between students' conceptions of sound attenuation in materials and the characteristics that they associate with acoustic behaviour of materials

\begin{tabular}{|c|c|c|c|c|c|}
\hline \multirow{2}{*}{ Property } & \multirow{2}{*}{$\begin{array}{l}\text { Conception } \\
\text { of sound } \\
\text { attenuation }\end{array}$} & \multicolumn{2}{|c|}{$\begin{array}{c}\text { Higher value of the property } \\
\text { implies more capacity for } \\
\text { attenuating sound }\end{array}$} & \multicolumn{2}{|c|}{$\begin{array}{l}\text { Higher value of the property } \\
\text { implies less capacity for } \\
\text { attenuating sound }\end{array}$} \\
\hline & & $\begin{array}{l}\text { Number } \\
\text { of } \\
\text { students }\end{array}$ & Students' quote & $\begin{array}{l}\text { Number } \\
\text { of } \\
\text { students }\end{array}$ & Students' quote \\
\hline \multirow{4}{*}{ Porosity } & C1 & - & & $\begin{array}{l}2 / 33 \\
(6 \%)\end{array}$ & $\begin{array}{l}\text { "Materials with low } \\
\text { porosity result in sound } \\
\text { waves colliding [against } \\
\text { them]" }\end{array}$ \\
\hline & $\mathrm{C} 2$ & $\begin{array}{c}9 / 33 \\
(27 \%)\end{array}$ & $\begin{array}{l}\text { "More porous materials } \\
\text { attenuate sound more } \\
\text { since sound waves spend } \\
\text { more energy when going } \\
\text { through changes of } \\
\text { medium" }\end{array}$ & - & \\
\hline & C3 & $\begin{array}{l}3 / 33 \\
(9 \%)\end{array}$ & $\begin{array}{l}\text { "[Within porous } \\
\text { materials] some } \\
\text { phenomena occur. For } \\
\text { instance, sound remains } \\
\text { inside the pores, and so } \\
\text { a great part of the sound } \\
\text { is not transmitted to the } \\
\text { opposite side" }\end{array}$ & - & \\
\hline & $\mathrm{C4}$ & $\begin{array}{l}3 / 33 \\
(9 \%)\end{array}$ & $\begin{array}{l}\text { "The more porous the } \\
\text { material, the better the } \\
\text { sound absorber it is" }\end{array}$ & $\begin{array}{l}2 / 33 \\
(6 \%)\end{array}$ & $\begin{array}{l}\text { "The less porous the } \\
\text { material, the better a } \\
\text { sound reflector it is" }\end{array}$ \\
\hline \multirow{2}{*}{ Density } & C1 & $\begin{array}{c}4 / 33 \\
(12 \%)\end{array}$ & $\begin{array}{l}\text { "More dense materials } \\
\text { result in sound waves } \\
\text { colliding [against } \\
\text { them]" }\end{array}$ & - & \\
\hline & $\mathrm{C} 2$ & $\begin{array}{c}4 / 33 \\
(12 \%)\end{array}$ & $\begin{array}{l}\text { "The denser a material, } \\
\text { the less vibration it } \\
\text { allows in its particles } \\
\text { and therefore it has }\end{array}$ & $\begin{array}{l}2 / 33 \\
(6 \%)\end{array}$ & $\begin{array}{l}\text { "If a material is denser, } \\
\text { its particles are closer } \\
\text { and therefore it transmits } \\
\text { sound better than a less }\end{array}$ \\
\hline
\end{tabular}




\begin{tabular}{|c|c|c|c|c|c|}
\hline & & & $\begin{array}{l}\text { more difficulty in } \\
\text { transmitting sound" }\end{array}$ & & dense material" \\
\hline & C3 & - & & - & \\
\hline & C4 & $\begin{array}{l}2 / 33 \\
(6 \%)\end{array}$ & $\begin{array}{l}\text { "The denser a material, } \\
\text { the more sound it } \\
\text { reflects" }\end{array}$ & $\begin{array}{l}1 / 33 \\
(3 \%)\end{array}$ & $\begin{array}{l}\text { "If the material has a low } \\
\text { density, with lots of } \\
\text { spaces between particles, } \\
\text { it absorbs sound a lot" }\end{array}$ \\
\hline \multirow[t]{4}{*}{$\begin{array}{l}\text { Distance } \\
\text { between } \\
\text { particles }\end{array}$} & C1 & - & & $\begin{array}{l}1 / 33 \\
(3 \%)\end{array}$ & $\begin{array}{l}\text { "Some materials have } \\
\text { their particles very } \\
\text { close and then air } \\
\text { particles cannot go } \\
\text { through them. In } \\
\text { contrast, if the } \\
\text { particles are more } \\
\text { separated and allow } \\
\text { air to enter the } \\
\text { material then the } \\
\text { vibrations will also } \\
\text { enter [the material] } \\
\text { and we can hear } \\
\text { sound" }\end{array}$ \\
\hline & $\mathrm{C2}$ & $\begin{array}{l}3 / 33 \\
(9 \%)\end{array}$ & $\begin{array}{l}\text { "If particles are more } \\
\text { separated, then the } \\
\text { vibration is transmitted } \\
\text { worse than in a material } \\
\text { in which the particles } \\
\text { are closer" }\end{array}$ & - & \\
\hline & C3 & - & & - & \\
\hline & C4 & $\begin{array}{l}1 / 33 \\
(3 \%)\end{array}$ & $\begin{array}{l}\text { "[If the material] } \\
\text { has a lot of space } \\
\text { between particles, it } \\
\text { absorbs sound a lot" }\end{array}$ & - & \\
\hline
\end{tabular}

As illustrated in Table 4, all students who consider that sound is attenuated by materials that act as obstacles which do not allow sound to go through them $(\mathrm{C} 1)$, also consider that sound attenuating materials are dense, not very porous and are formed by closer particles. On the other hand, students who state that sound attenuation in materials is produced when sound remains inside the material and does not get out (C3) believe that sound attenuating materials are porous. In this sense, we interpret that the acoustic role that students attribute to certain properties or the internal structure of materials is rather coherent with their conception of sound attenuation.

Concerning students who conceive sound attenuation as a phenomenon occurring while sound propagates within the material (C2), we evidence some differences in the roles that these students attribute to density. While some students consider that sound attenuating materials need to be dense so that their particles are difficult to move, other students state that sound attenuating materials should not be dense so that its particles are very separated from each other, and so they have more difficulty in transmitting sound. This last statement was also reported by Linder (1993), who found that many students consider that the density of materials uniquely depends on the distance between its particles, but not on the mass and packaging of particles.

Furthermore, we found that students use indistinctively specific properties of materials to describe them. In this way, several students do not only associate density with close proximity between particles, but they also consider that the terms dense, non-porous, and compact are equivalent.

\section{Conclusions}

The findings of this study provide evidence that students, who had been previously taught about the nature and propagation of sound and had measured the capacity for attenuating sound of different materials during a laboratory session, but who had not received any prior instruction on sound attenuation, were already able to elaborate on different types of - naïve or reasonable - explanations of sound attenuation. That is to say, there is evidence that students built some models of sound attenuation 
without explicit teaching on this topic, just having experience with materials with different acoustic absorption capacity.

Regarding the first research question, we could conclude that about one quarter of students conceptualise sound attenuating materials as sound barriers avoiding the passage of sound, and thus reflection is considered the only mechanism for sound attenuation. A smaller number of students recognise absorption as a mechanism of sound attenuation within a material. In any case, most of the students conceive the phenomenon of sound attenuation as the difficulty in transmitting sound in a material. In some cases, attenuation is conceived as being accompanied by the modification of the characteristics of sound waves. Remarkably, a small number of these students were able to relate sound attenuation to energy decrease.

The results of this study also provide evidence that students' preconceptions of sound attenuation reveal their conceptions of the nature and propagation of sound. In this sense, some students' responses show that the most common alternative conception is of sound as a physical substance that can go through a material or not depending on certain characteristics of the material. This conception corresponds to the well known "entity model of sound" described by Hrepic et al. (2010).

With regards to the second research question, the results of this study indicate that the role of the properties of a material and the role of the internal structure that students associate with its acoustic behaviour depend on their conceptions of sound attenuation. That is to say, the same material property can be conceived as a facilitator or an obstacle to absorb or to reflect sound, depending on the student's model of sound attenuation. Accordingly, the best sound attenuating materials are considered dense and non-porous by those students who think that sound attenuation is the result of the non-entrance of sound in a material. Sound attenuating materials are then conceived as sound barriers.

Furthermore, the findings also illustrate a lack of differentiation between certain properties of materials, such as density, compactness or porosity. The non-discrimination between certain scientific terms is a recurrent issue that needs plenty of attention.

Finally, the results of the present study also support previous research findings such as those reported by Linder (1993) concerning the oversimplification of the relationship between the density of materials and their microstructure (i.e. "the smaller the distance between particles, the denser the material is").

From this study, we conclude that students have a variety of preconceptions about sound attenuation and of the acoustic properties of materials, although some of them are more coherent from a scientific point of view than others. Thus, this research study supports the fact that students do not develop conceptual models that allow them to appropriately explain sound attenuation in materials as a mere result of their active engagement in designing and performing experiments in the REVIR session. The REVIR scenario, however, turned out to be a favourable context for allowing students to enrich and elicit their initial explanations.

\section{Implications for design}

Within the research-based design paradigm, this study is relevant not only because it contributes to clarifying students' conceptions about sound attenuation and of the acoustic properties of materials, but also because the findings can be used to adjust and support the decisions made when designing a teaching sequence, like those already developed by Pintó et al. (2009) on the Acoustic Properties of Materials.

What benefits could there be of a teaching sequence on the Acoustic Properties of Materials?

Sound is an area of physics widely considered an essential component of most official science curricula. A traditional approach to the study of sound in secondary schools usually includes a macroscopic description of mechanical longitudinal waves in terms of its main characteristics (amplitude, frequency, etc.), and a microscopic description of the mechanisms involved in sound production, propagation and detection, such as vibration and the interaction between the particles of the medium.

Moreover, we can find relevant socioscientific issues related to sound, such as why we should avoid listening to and/or emitting loud music. Some authors (Sadler 2009) plead for the discussion of these issues in school science lessons in order to promote learning experiences that can affect students' thinking and the development of scientific literacy. In fact, linking sound with noise pollution has also become more common in curricula with an STS (Science-Technology-Society) or environmental education 
approach. To make students aware of this problem and more able to select the appropriate materials for practical soundproofing tasks, we designed a teaching/learning sequence drawn upon the findings presented here. The teaching sequence allows engaging students in the analysis of the relationship between the properties and internal structure of specific materials so that they are able to account for their acoustic behaviour. This meant tackling the study of sound from the perspective of Materials Science, emphasising the selection, testing and characterisation of the appropriate materials for noise control. This perspective is in agreement with Wendell and Lee's (2010) view, who consider that certain practices common to Materials Science, such as the characterisation of materials by intensive properties, identification of the properties needed for particular purposes, and selection of existing materials with desirable properties, play an important role in young students' physical science learning.

What have we learned from this study about the teaching of this topic that could contribute to the design of a TLS on APM?

According to Scott, Asoko and Leach (2007), insights into how to teach conceptual content will only arise through design research, where insights into domain-specific reasoning are drawn upon in the design of teaching materials, which are then tested and developed in an iterative process (Lijnse 1995). According to this, we made detailed use of the above-described students' preconceptions to the design of certain activities of the TLS on APM. Those activities are intended to facilitate students' eliciting of their own conceptions and make them yearn for a need for theoretical elements to justify their statements.

Considering the differences between the content to be taught (Hernández et al. 2011) and the students' conceptions evidenced in this study, we could establish the learning demands (Leach \& Scott 2002) for 15-16 year-old students. These learning demands assume that the students involved come to:

- Understand the nature and propagation of sound as an event or process instead of as an entity.

- Conceive sound attenuation as a process of energy dissipation that involves reflection and absorption rather than an effect caused by materials when "hindering the entrance of sound" or "capturing sound".

- Appropriately conceptualize the acoustic properties of materials, at both macroscopic and microscopic levels, and relate them to the acoustic behaviour of materials. For instance, this would imply considering the density of solid materials as being related to the mass and packaging of their particles instead of associating it with the distance between particles.

Finally, the research study also gave us some insight into how to organise the content to be taught in the TLS on APM in order to facilitate students' development of a more coherent conceptual framework:

- Since students' conceptions of sound attenuation are influenced by their conceptions of the nature and propagation of sound, teaching about sound waves and their propagation should be a prerequisite of the TLS on APM.

- Similarly, since the properties and internal structure that students associate with the acoustic behaviour of materials is often based on their conceptions of sound attenuation in materials, we decided to develop their conceptions by distinguishing the mechanism of sound reflection from that of sound absorption. In this way, students would identify two types of sound attenuating materials (sound reflectors and sound absorbers) and would attribute different acoustic properties to them.

- As students tend to have a simplistic view of the relationship between properties and internal structure of materials, we decided to explicitly tackle these relationships by developing tasks and analogies to unambiguously develop students' understanding of the so-called acoustic properties of materials.

\section{Acknowledgments}

This work was funded by the Consell Social of the Universitat Autònoma de Barcelona in the REVIR project. M. I. Hernández was supported by the Ministry of Science and Innovation (MICINN) under the FPU programme.

\section{References}

Driver, R., Squires, A., Rushworth, P., \& Wood-Robinson, V. (1994). Making sense of secondary science: Research into children's ideas. London: Routledge.

Eshach, H., \& Schwartz, J. (2006). Sound stuff? Naïve materialism in middle-school students' conceptions of sound. International Journal of Science Education, 28(7), 733-764.

Hernández, M. I., Couso, D., \& Pintó, R. (2011). Teaching acoustic properties of materials in secondary school: testing sound insulators. Physics Education, 46(5), 559-569. 
Hrepic, Z., Zollman, D. A., \& Rebello, N. S. (2010). Identifying students' mental models of sound propagation: The role of conceptual blending in understanding conceptual change. Physical Review Special Topics - Physics Education Research, 6, 1-18.

Lawrence, I. (2008). Sounding off and lighting up. Physics Education, 43(1), 62-67.

Leach, J., \& Scott, P. (2002). Designing and evaluating science teaching sequences: An approach drawing upon the concept of learning demand and a social constructivist perspective on learning. Studies in Science Education, 38, 115-142.

Lijnse, P. (1995). "Developmental research" as a way to an empirically based "didactical structure" of science. Science Education, 79(2), 189-199.

Linder, C. J. (1992). Understanding sound: So what is the problem? Physics Education, 27, 258-264.

Linder, C. J. (1993). University physics students' conceptualizations of factors affecting the speed of sound propagation. International Journal of Science Education, 15(6), 655-662.

Marton, F. (1981). Phenomenography - Describing conceptions of the world around us. Instructional Science, 10, 177-200.

Maurines, L. (1993). Spontaneous reasoning on the propagation of sound. Paper presented at the Third International Seminar on Misconceptions and Educational Strategies in Science and Mathematics, Ithaca, NY.

Mazens, K., Lautrey, J. (2003). Conceptual change in physics: children's naive representations of sound. Cognitive Development, 18, 159-176.

Pintó, R., Couso, D., Hernández, M. I., Armengol, M., Cortijo, C., Martos, R., et al. (2009). Acoustic properties of materials: Teachers' manual \& teaching and learning activities. Nicosia: University of Cyprus.

Pintó, R., Couso, D., \& Hernández, M.I. (2010). An inquiry-oriented approach for making the best use of ICT in the classroom. eLearning Papers, 20, 1-14.

Sadler, T. D. (2009). Socioscientific issues in science education: labels, reasoning, and transfer. Cultural Studies of Science Education, 4, 697-703.

Scott, P., Asoko, H., \& Leach, J. (2007). Student conceptions and conceptual learning in science. In S. K. Abell \& N. G. Lederman (Eds.), Handbook of Research on Science Education (pp. 31-56). Mahwah, NJ: Lawrence Erlbaum Associates, Routledge Publishers.

Taber, K. S. (2000). Multiple frameworks? Evidence of manifold conceptions in individual cognitive structure. International Journal of Science Education, 22(4), 399-417.

Wendell, K. B., \& Lee, H.-S. (2010). Elementary students' Learning of materials science practices through instruction based on engineering design tasks. Journal of Science Education and Technology, 19, 580-601.

Wittmann, M., Steinberg, R., \& Redish, E. (2003). Understanding and affecting student reasoning about sound waves. International Journal of Science Education, 25(8), 991-1013. 